Pour les patients avec une consommation d'alcool à risque, le médecin de famille est un interlocuteur privilégié pour autant qu'il ose et sache comment aborder le sujet. Le premier guide pratique visant à soutenir les médecins dans cette exigeante démarche a été élaboré il y a plus de 10 ans dans le cadre de la campagne de prévention "Alcool, ça débouche sur quoi?». Depuis, les options thérapeutiques se sont nettement améliorées et c'est pourquoi les auteurs ont heureusement décidé de publier une deuxième édition augmentée qui vous est présentée dans I'article ci-après.

Dr Christine Romann, membre du Comité central de la FMH, responsable du département Promotion de la santé et prévention

\title{
Interventions brèves pour traiter l'alcoolodépendance
}

\section{Pierre Loeb}

Dr, spécialiste en médecine générale, Bâle

\section{Pourquoi évite-t-on d'aborder les problèmes d'alcool en consultation?}

Le sujet est délicat, d'une part parce que le taux de réussite a été très modeste jusqu'à présent et, de l'autre, parce qu'un médecin traitant ne saurait exiger de son patient qu'il se soumette à un traitement ou suive une cure de désintoxication. Bien que cette affirmation puisse paraître exagérée, les médecins disposaient jusqu'à présent d'options thérapeutiques limitées ou n'étaient parfois pas très convaincus par le principe d'abstinence ou les autres formes de consommation contrôlée prônées par le guide sur la consommation d'alcool. Des recommandations telles que «Buvez un peu moins, ne commencez à boire que le soir, ne buvez pas plus de trois unités d'alcool par jour, buvez toujours de l'eau d'abord, essayez la bière sans alcool, instaurez une journée sans alcool, ne buvez pas seul, ne buvez pas chez vous, etc.» étaient certes bien intentionnées, mais aboutissaient rarement à moyen et long terme. Et les résultats n'étaient guère meilleurs en jouant de notre autorité: «Vos gamma-GT et vos valeurs hépatiques ont à nouveau augmenté, cela se voit à l'échographie...» A cela s'ajoutait la difficulté à aborder un sujet délicat qui risquait de mettre à mal la relation avec nos patients. De plus, pour être franc, nous aimons aussi parfois «boire un verre», un plaisir qui, chez nos patients, ne se limite peut-être pas toujours à "un verre». Or si nous voulons quand même aborder le sujet, cela exige de prendre le temps et aussi le risque d'ouvrir la boîte de Pandore. Addiction rime en effet souvent avec problèmes sexuels, difficultés professionnelles, crise de couple, violences domestiques, problèmes financiers.
Autant de raisons qui font que l'on préfère éviter le sujet.

Il y a dix ans, l'Office fédéral de la santé publique lançait sa première campagne de prévention intitulée "Alcool, ça débouche sur quoi?» qui ciblait principalement les jeunes consommateurs à risque. Au moyen de phrases et d'images simples à retenir (vous vous souvenez sans doute des verres de whisky brisés), le groupe de travail médical qui accompagnait le projet avait organisé des ateliers destinés aux médecins de famille afin de les aider à détecter les personnes à risque - dont la consommation n'était pas de prime abord considérée comme pathologique ou exagérée et leur permettre d'aborder la question de front à l'aide de données comparatives claires pour qu'ils puissent déterminer avec leur patient ce que trop veut dire. La première édition du guide pratique a été publiée en complément à ces ateliers afin de permettre aux médecins d'approfondir seuls la question. Ce guide offrait aux médecins des outils pour une meilleure détection et sélection des patients et les familiarisait à la méthode de l'intervention brève pour ouvrir le dialogue et engager les premières étapes du traitement. En revanche, les patients alcoolodépendants chroniques pour lesquels seules la désintoxication et l'abstinence entraient en ligne de compte ne figuraient pas dans ce concept thérapeutique.

\section{Seconde édition revue et augmentée}

Qu'est-ce qui a changé au cours de la dernière décennie pour inciter les auteurs à publier une nouvelle version revue et augmentée du guide de pratique? Les changements se résument en trois points. 
